\title{
PROJECTIVE VARIETIES OF LOW CODIMENSION IN CHARACTERISTIC $p>0$
}

BY

\author{
ROBERT SPEISER
}

\begin{abstract}
Let $X$ be an s-dimensional closed Cohen-Macaulay subvariety of projective $n$-space, over an algebraically closed field of characteristic $p>0$. Assume $s>\frac{1}{2}(n+1)$. Then (1) every stratified vector bundle on $X$ is trivial; (2) $X$ is simply connected. Assertion (1) generalizes Gieseker's result for projective space, while (2) is a strengthened analogue of results of Barth and Ogus in characteristic zero.
\end{abstract}

0. Introduction. In the last few years it has become increasingly evident that varieties of low codimension in $\mathbf{P}_{n}$, if smooth enough, share (as do divisors) many important geometric properties with $\mathbf{P}_{n}$ itself. This conception is pursued systematically in a recent exposition by Hartshorne [8], in which most of the results obtained by the time of his writing are reviewed. The purpose of the present paper is to contribute further statements of this kind. To be specific, let us fix an algebraically closed base field $k$, let $Y \subset \mathbf{P}_{n}=$ $\mathbf{P}_{n}(k)$ be a closed s-dimensional local complete intersection subvariety, and consider some possibilities:

(0.1) Barth theorems for cohomology. Let $H^{*}$ denote some reasonable cohomology theory. (In characteristic 0 we could take algebraic De Rham cohomology, or, if $k=\mathrm{C}$, any of the classical theories, say with coefficients in $\mathbf{Z}$ or $\mathbf{C}$.) Then the natural maps

$$
H^{i}\left(\mathbf{P}_{n}\right) \rightarrow H^{i}(Y)
$$

are isomorphisms, for $i \leqslant 2 s-n$.

(0.2) Barth theorems for $\pi_{1}$ : if $s \geqslant \frac{1}{2}(n+1)$, then $Y$ is simply connected.

(0.3) Triviality of stratified bundles (or equivalently, of bundles with integrable connection, if $\operatorname{char}(k)=0)$. If $s>\frac{1}{2}(n+1)$, then every stratified vector bundle on $Y$ is trivial.

(0.4) Hartshorne's conjecture. If $Y$ is smooth, and if $s>(2 / 3) n$, then $Y$ is a global complete intersection in $\mathbf{P}_{n}$.

For $Y$ smooth, $k=\mathrm{C}$, with singular cohomology with coefficients in $\mathbf{C}$, (0.1) is due to Barth, and (0.2) is due to Barth and Larsen (cf. [2], [3]). For $k$ any algebraically closed field in characteristic $0,(0.1),(0.2)$ and $(0.3)$ were

Received by the editors January 4, 1977.

AMS (MOS) subject classifications (1970). Primary 14F05, 14E20; Secondary 14B10.

- American Mathematical Society 1978 
proved by Ogus, with algebraic De Rham cohomology in (0.1). (Cf. [11], [12].) In Ogus' versions, $Y$ can have singularities, and then one has progressively weaker statements as the singularity of $Y$ increases.

In characteristic $p>0,(0.1)$ was treated in complete generality in joint work of Hartshorne and the author [10], with Z/pZ-étale cohomology. Our results were analogous to those of Ogus. In this paper we establish (0.2) and $(0.3)$ for $Y$ Cohen-Macaulay, ${ }^{1}$ also in characteristic $p$.

Thus (0.4) remains open, in any characteristic. We mention it here because, via the Lefschetz theorems, it motivates the other statements. These, in turn, suggest that (0.4) itself, or something like it, "ought" to be true.

We will establish our versions of $(0.2)$ and $(0.3)$ in the following logical order:

$$
\text { general principles } \Rightarrow(0.3) \Rightarrow(0.2) \text {. }
$$

Since the method by which we intend to show that stratified bundles are trivial will ultimately determine which "general principles" we intend to refer to, $\S 1$ is devoted to a discussion of stratified bundles. We go back to Grothendieck's original definition [5, p. 357] in terms of "formal descent data." We then give a criterion under which the "formal descent data" can be extended to genuine descent data, so that the bundle in question will descend to $\operatorname{Spec}(k)$, and hence must be trivial. (Our criterion is that the diagonal

$$
\Delta(Y) \subset Y \times Y
$$

is sufficiently "ample", almost that $\operatorname{Lef}(Y \times Y, \Delta(Y))$ should hold.)

To achieve this extension when $Y$ has low codimension we study the formal completion of $\Delta\left(\mathbf{P}_{n}\right)$ in $\mathbf{P}_{n} \times \mathbf{P}_{n}$ first, in §2. We show that this subvariety has just about all the "ampleness" one might reasonably want. Then in $\$ 3$ we take up (0.3) for general $Y$. We have already observed that the diagonal embedding

$$
\Delta\left(\mathbf{P}_{n}\right) \subset \mathbf{P}_{n} \times \mathbf{P}_{n}
$$

is quite "ample". Since

$$
\Delta(Y)=\Delta\left(\mathbf{P}_{n}\right) \cap Y \times Y
$$

we can then ask whether some measure of the "ampleness" of the first embedding is preserved under intersection with $Y \times Y$, if the codimension of $Y$ in $P_{n}$ is low. The "intersection theorem" (3.1.2) answers this question in the affirmative, so we obtain (0.3).

Finally in $\S 4$ we show that $(0.3) \Rightarrow(0.2)$. This result is actually folklore: if $f$ :

\footnotetext{
${ }^{1}$ An independent proof of $(0.2)$ using the full force of the local theory appears in [10, 84]. The present proof is purely global and proceeds from an entirely different idea. In particular we obtain (0.3) for $Y$ Cohen-Macaulay whereas the results of [10] would only imply $(0.3)$ for $Y$ smooth, at best. (Compare Katz' result [4, Theorem 1.3].)
} 
$Y_{1} \rightarrow Y$ is an étale cover, $f_{*}\left(O_{Y_{1}}\right)$ is a stratified bundle on $Y$, which, if trivial, implies the triviality of the covering.

Readers of recent literature on stratifications (for example [4]) will perhaps be surprised by our approach, in that differential operators are never considered, and the perhaps tempting analogy with integrable connections in characteristic 0 is not exploited. We give our definitions purely in terms of descent theory, and stick to this consistently throughout. A particular advantage of this approach is that we can avoid tedious explicit calculations with differentials, basing our work instead on conceptual, geometric ideas, in particular on the "intersection theory" of $\$ 3$.

Since giving an integrable connection in characteristic zero is tantamount - to giving a stratification, our work extends the results of Ogus ([11], [12]) to the case of arbitrary characteristic, but out technique of reducing to the diagonal is completely different from his.

This difference in methodology seems to be necessary if one wishes to have the strongest possible results. A peculiar feature of our theory is that the main statements about the "ampleness" of $\Delta$ hold only in characteristic $p>0$, but, in compensation, our results are stronger: $(0.2)$ and $(0.3)$ are valid for $Y$ Cohen-Macaulay, whereas Ogus' hold only for local complete intersections.

This work has benefited considerably from the generosity of colleagues. $\mathbf{R}$. Hoobler first taught me about stratifications, and called my attention to Gieseker's work. Once the method of $\$ 1$ dawned on me, it took some time to crystallize the theory in \$2. Here I am indebted to A. Ogus and R. Hartshorne for some helpful discussions. Were it not, however, for the joint work with Hartshorne on the local theory [10], the ideas leading to this paper might never have reached fruition. I therefore thank him, especially, for his inspiration and example.

Notations and preliminaries. The letter " $k$ " will always denote an algebraically closed field, and " $p$ " will always denote a prime rational integer. We work in the category of separated schemes of finite type over $\operatorname{Spec}(k)$, each equipped with the Zariski topology. A reduced and irreducible such scheme will be called a variety.

We shall assume that the reader has a general familiarity with Hartshorne's theory of "ample subvarieties", as developed in [9], to which we shall refer again and again. In particular, for any scheme $X$, the cohomological dimension of $X$ will be the integer

$$
\operatorname{cd}(X)=\inf \left\{i \mid H^{j}(X, F)=0 \text { for all coherent sheaves } F \text { on } X \text {, all } j>i\right\} .
$$

Finally, we sometimes use the term "vector bundle" for a locally free sheaf of finite, constant rank, and similarly "line bundle" for an invertible sheaf. 
1. Stratified vector bundles. We review Grothendieck's original definition of a stratification and compare this with descent data. Using faithfully flat descent we give conditions under which all stratified vector bundles on a given base space are trivial.

(1.1) Let $X$ be an $S$-scheme, for a base scheme $S$, and assume $X$ is separated over $S$. Form the products

$$
X \stackrel{p_{1}}{\leftarrow} X \times{ }_{S} X \stackrel{p_{2}}{\rightarrow} X
$$

and

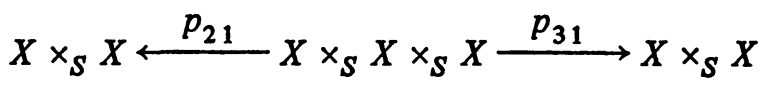

$$
\begin{aligned}
& \downarrow p_{32} \\
& X \times{ }_{S} X
\end{aligned}
$$

over $S$, where in the triple product $p_{i j}$ is the projection onto the $i$ th and $j$ th factors.

Denote by $\Delta$ the diagonal subscheme in $X \times_{s} X$, by $\Delta^{\prime}$ the diagonal subscheme in $X \times{ }_{s} X \times_{s} X$. Since $X$ is separated over $S$, these subschemes are closed.

Let $\Delta_{(r)} \subset X \times_{s} X$ be the $r$ th infinitesimal neighborhood of $\Delta$, and let $\Delta_{(r)}^{\prime} \subset X \times{ }_{s} X \times_{s} X$ be the analogous neighborhood of $\Delta^{\prime}$. We obtain projections

$$
X \stackrel{q(r)}{\leftarrow} \Delta_{(r)} \stackrel{q 2^{(r)}}{\rightarrow} X
$$

and

from the previous diagrams.

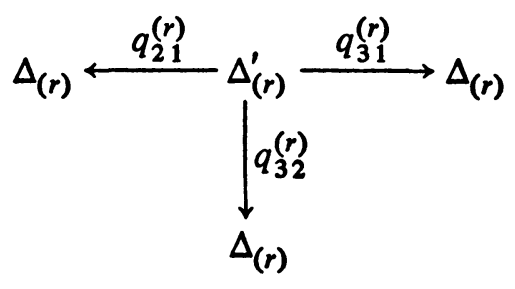

Finally, let $F$ be a quasicoherent sheaf on $X$.

(1.2) A stratification on $F$ (relative to $S$ ) is a compatible system of isomorphisms

$$
\theta_{r}: q_{1}^{(r)^{*}}(F) \stackrel{\sim}{\rightarrow} q_{2}^{(r)^{*}}(F)
$$

over $\Delta_{(r)}$ for all $r>1$, such that the cocycle condition

$$
q_{31}^{(r)^{*}}(\theta)=q_{32}^{(r) *}(\theta) \circ q_{21}^{(r) *}(\theta)
$$

holds on $\Delta_{(r)}^{\prime}$. (Compare [5, p. 356].) 
(1.3) If $X$ is the formal completion of $X \times_{s} X$ along $\Delta$, and $X^{\prime}$ is the formal completion of $X \times_{s} X \times_{s} X$ along $\Delta^{\prime}$, we have projections

$$
X \stackrel{q_{1}}{\longleftarrow} \stackrel{q_{2}}{\longrightarrow} X
$$

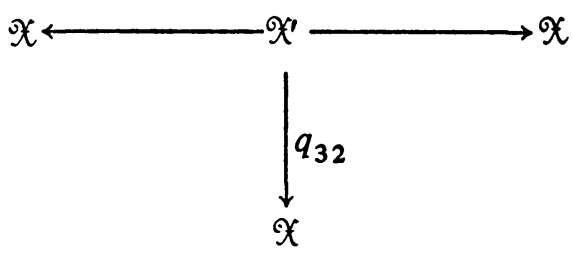

and a stratification on $F$ induces an isomorphism

$$
\hat{\theta}: q_{1}^{*}(F) \rightarrow q_{2}^{*}(F) \quad(\text { on } \mathscr{X})
$$

such that the cocycle condition

holds on $X^{\prime}$.

$$
q_{31}^{*}(\hat{\theta})=q_{32}^{*}(\hat{\theta}) \circ q_{21}^{*}(\hat{\theta})
$$

(1.4) The preceding can be thought of as "formal descent data" for $F$. It is worth comparing with real descent data:

By descent data for $F$ (relative to $S$ ) we mean an isomorphism

$$
\varphi: p_{1}^{*}(F) \stackrel{\sim}{\rightarrow} p_{2}^{*}(F)
$$

over $X \times_{S} X$ such that the cocycle condition

$$
p_{31}^{*}(\varphi)=p_{32}^{*}(\varphi) \circ p_{21}^{*}(\varphi)
$$

holds, over $X \times{ }_{s} X \times{ }_{s} X$.

From Grothendieck's theory of descent we know that if $X$ is faithfully flat over $S$ (e.g., if $S=\operatorname{Spec}(k)$ for a field $k$ ), then $F$ has descent data relative to $S$ if and only if $F$ descends to $S$, i.e., $F=p^{*}(E)$ for a quasicoherent sheaf $E$ on $S$, where

is the projection [6, VIII.1].

$$
p: X \rightarrow S
$$

(1.5) For a scheme (or, more generally, formal scheme), $X$, denote by $\mathcal{L F}(X)$ the category of locally free sheaves on $X$. If $Y \subset X$ is a closed subscheme, denote by $\hat{X}$ the formal completion of $X$ along $Y$, and by $\mathcal{G} \mathcal{L F}(Y)$ the category of germs of locally free sheaves on neighborhoods of $Y$ in $X$.

We obtain a functor

$$
\mathfrak{:} \mathcal{G} \mathscr{F}(Y) \rightarrow \mathfrak{L F} F(\hat{X})
$$

which associates to each locally free sheaf $F$ in a neighborhood of $Y$ its formal completion $\hat{F}$ on $\hat{X}$. 
We shall say that the Lefschetz condition $\operatorname{Lef}(X, Y)$ holds if the completion function $\mathcal{G} \mathscr{L} \mathscr{F}(Y) \rightarrow \mathscr{L} \mathscr{F}(\hat{X})$ is fully faithful. (Compare [7, p. 112], [9, p. 164].)

(1.6) This "weak" Lefschetz condition should be compared with the effective Lefschetz condition Lef $f(X, Y)[7$, loc. cit.] which is said to hold if $\mathcal{G} \mathfrak{L} \mathscr{F}(Y) \rightarrow \mathscr{L} \mathscr{F}(\hat{X})$ is an equivalence of categories.

(1.7) Another variation on $\operatorname{Lef}(X, Y)$ runs as follows: we shall say that $S(X, Y)$ holds if the completion functor $\mathcal{L F}(X) \rightarrow \mathfrak{L F}(\hat{X})$ is fully faithful, or, equivalently, if for every locally free sheaf $F$ on $X$ the natural map

$$
H^{0}(X, F) \rightarrow H^{0}(\hat{X}, \hat{F})
$$

is bijective.

Here is our main result:

THEOREM (1.7.1) Let $k$ be a field, $X$ a connected, separated, locally noetherian scheme of finite type over $\operatorname{Spec}(k)$. Denote by $X \times X$ the product over $\operatorname{Spec}(k)$ and by $\Delta \subset X \times X$ the diagonal. If $S(X \times X, \Delta)$ holds, then every stratified vector bundle on $X$ is trivial.

Proof. Let a vector bundle $F$ on $X$ have a stratification. We obtain an induced isomorphism ("formal descent data")

$$
\hat{\boldsymbol{\theta}}: q_{1}^{*}(F) \rightarrow q_{2}^{*}(F) \text {. }
$$

Since $q_{i}^{*}(F)(i=1,2)$ lies in the image of ", it follows from $S(X \times X, \Delta)$ that $\hat{\theta}$ is induced by a unique bijection

$$
\varphi: p_{1}^{*}(F) \rightarrow p_{2}^{*}(F)
$$

over $X \times X$. Hence we need only verify the cocycle condition for $\varphi$ in order to have descent data for $F$, relative to the projection

$$
p: X \rightarrow \operatorname{Spec}(k) \text {. }
$$

Now along the diagonal $\Delta \subset X \times X \times X$, completing any vector bundle $G$ on $X \times X \times X$ yields an injection

$$
H^{0}(X \times X \times X, G) \rightarrow H^{0}(\widehat{X \times X \times X}, \hat{G}),
$$

since $X \times X \times X$ is connected and locally noetherian. Hence the cocycle condition for $\varphi$, viewed as a relation between two sections of a vector bundle $G$, holds if its completion holds in $\hat{G}$. But we obviously have the cocycle condition for $\hat{\theta}$, so it follows that we have it for $\varphi$ as well.

Since we have descent data for $F$, and since the projection $p$ is faithfully flat, the main theorem of faithfully flat descent [6, VIII, Theorem 1.1] implies that $F$ descends to $\operatorname{Spec}(k)$, that is,

$$
F=\theta_{X} \otimes_{k} V,
$$


where $V$ is a finite dimensional $k$-vector space. Thus $F$ is trivial, and our proof is complete.

REMARK. In the proof it would be enough to verify that $\hat{\theta}$ is in the image of ' in order to assure ourselves that $F$ is trivial. have

COROLlary (1.7.2) Let $X$ be smooth and proper over $\operatorname{Spec}(k)$. Suppose we have

$$
\operatorname{cd}(X \times X-\Delta) \leq 2 \cdot \operatorname{dim}(X)-2 .
$$

Then every stratified vector bundle on $X$ is trivial.

Proof. By [15, Theorem 2], we know that $S(X \times X, \Delta)$ holds.

2. The diagonal in $\mathbf{P}_{n} \times \mathbf{P}_{n}$. After preliminaries (2.1) we consider the diagonal $\Delta \subset \mathbf{P}_{n} \times \mathbf{P}_{n}$ in (2.2). It turns out that $\operatorname{cd}\left(\mathbf{P}_{n} \times \mathbf{P}_{n}-\Delta\right)$ is small-as we shall need for our applications-only in characteristic $p>0$. The consequences we shall later use, valid solely in characteristic $p$, are explained in (2.3).

(2.1) We first collect some facts we shall need later concerning the geometry of $\mathbf{P}_{n} \times \mathbf{P}_{n}$.

Denote by $\theta(\nu)$ the standard invertible sheaf on $\mathbf{P}_{n}$. Set

$$
\theta(\mu, \nu)=p_{1}^{*}(\theta(\mu)) \otimes p_{2}^{*}(\theta(\nu))
$$

in $\operatorname{Pic}\left(\mathbf{P}_{n} \times \mathbf{P}_{n}\right)$. Then $(\mu, \nu) \rightarrow \Theta(\mu, \nu)$ defines a group isomorphism

$$
\mathbf{Z} \oplus \mathbf{Z} \rightarrow \operatorname{Pic}\left(\mathbf{P}_{n} \times \mathbf{P}_{n}\right) \text {. }
$$

Note also that $\theta(\mu, \nu)$ is very ample if and only if $\mu$ and $\nu$ are both $>0$. (One obtains then the Segre embedding, cf. [EGA II, 4.3].)

Given a divisor $D$ on $\mathbf{P}_{n} \times \mathbf{P}_{n}$ we shall say $D$ is symmetric if $D$ is invariant under the automorphism $\sigma(x, y)=(y, x)$ of $\mathbf{P}_{n} \times \mathbf{P}_{n}$. Note that if $D$ is both effective and symmetric, then $D$ is very ample, and its divisor class is given by $\theta(d, d)$, with $d>0$.

Proposition (2.1.1). The diagonal $\Delta \subset \mathbf{P}_{n} \times \mathbf{P}_{n}$ is the set-theoretic intersection of $2 n-1$ very ample divisors.

Proof. For $n=1, \Delta$ is itself a very ample divisor. Hence assume $n>2$. For each linear subspace $W \subset \mathbf{P}^{n}$ of codimension 2, define a divisor $D_{W}$ on $\mathbf{P}_{n} \times \mathbf{P}_{n}$ by

$$
D_{W}=\left\{(x, y) \in \mathbf{P}_{n} \times \mathbf{P}_{n} \mid x, y \text { and } W \text { are contained in some hyperplane }\right\} \text {. }
$$

This $D_{W}$ is a symmetric, hence very ample divisor, and clearly $\Delta \subset D_{W}$. I claim that if $W_{1}, \ldots, W_{2 n-1}$ are sufficiently general linear subspaces of codimension 2, then $\cap D_{W_{l}}=\Delta$. Indeed, if $x \neq y$, let $L$ be the line in $\mathbf{P}_{n}$ spanned by $x$ and $y$. Clearly $(x, y) \in D_{W}$ if and only if $L$ meets $W$. But for 
sufficiently general $W_{i}$, I claim no line in $\mathbf{P}_{n}$ meets all of them. To verify this, consider the Grassmannian $G_{1, n}$ parametrizing the lines in $\mathbf{P}_{n}$. The condition that a line in $\mathbf{P}_{n}$ meet a subspace $W$ of codimension 2 in $\mathbf{P}_{n}$ is represented by a hyperplane section $H$ of $G_{1, n}$ in its Plücker embedding. Under the natural action of SL $(n+1)$ on the Grassmanian, $2 n-1$ sufficiently general translates of $H$ will have an empty intersection; indeed, the dimension of $G_{1, n}$ is $2 n-2$. Since each of these translates represents the condition that a given line meet the corresponding translate of $W$ on $\mathbf{P}_{n}$, it is clear how to choose the $W_{i}$ so that no line can meet them all. This proves the proposition.

REMARK. The discussion above embodies a substantial simplification suggested by the referee.

(2.2) Our main result here is the following.

THEOREM (2.2.1) Consider the diagonal subscheme $\Delta \subset \mathbf{P}_{n} \times \mathbf{P}_{n}$. Then

(a) $\operatorname{cd}\left(\mathbf{P}_{n} \times \mathbf{P}_{n}-\Delta\right)<2 n-2$;

(b) in characteristic 0 we have equality in (a);

(c) in characteristic $p>0$ we have $\operatorname{cd}\left(\mathbf{P}_{n} \times \mathbf{P}_{n}-\Delta\right)=n-1$.

Proof. By (2.1.1), $\mathbf{P}_{n} \times \mathbf{P}_{n}-\Delta$ is the union of $2 n-1$ affine open subsets. Hence we can compute cohomology on the resulting Čech $(2 n-2)$-simplex, which yields (a).

Assume now the characteristic is 0 . Then (b) is a direct consequence of Hartshorne's result [9, Theorem 8.6, p. 160], which asserts in our case that $\operatorname{cd}\left(\mathbf{P}_{n} \times \mathbf{P}_{n}-\Delta\right)<r$ implies the induced maps

$$
H_{D R}^{i}\left(\mathbf{P}_{n} \times \mathbf{P}_{n}\right) \stackrel{\alpha_{i}}{\rightarrow} H_{D R}^{i}(\Delta)
$$

on algebraic De Rham cohomology are bijective for $i<2 n-r$ and injective for $i=2 n-r$. Here $\alpha_{0}$ and $\alpha_{1}$ are bijective but $\alpha_{2}$ is not injective, hence $\operatorname{cd}\left(\mathbf{P}_{n} \times \mathbf{P}_{n}-\Delta\right)>n-2$. By (a), equality holds, so (b) is true.

Next let the characteristic be $p>0$, and consider (c). Since $\mathbf{P}_{n} \times \mathbf{P}_{n}-\Delta$ contains smooth complete varieties of dimension $n-1$ (e.g. $\{P\} \times H$ where $P$ is a point of $\mathbf{P}_{n}$ not in a hyperplane $H$ ) it follows easily from [9, Corollary 3.5 , p. 98] that $\operatorname{cd}\left(\mathbf{P}_{n} \times \mathbf{P}_{n}-\Delta\right) \geqslant n-1$. To obtain the reverse inequality, combine (a) and [14, Lemma, p. 147]: it will suffice to show that we have

$$
H^{i}\left(\mathbf{P}_{n} \hat{\times} \mathbf{P}_{n}, \hat{\theta}(\nu, \nu)\right)=0 \quad(i=1, \ldots, n-1)
$$

for $\nu \gg 0$, where "“" denotes formal completion along $\Delta$.

Let $I \subset \theta_{\mathbf{P}_{n} \times \mathbf{P}_{n}}$ by the ideal sheaf defining $\Delta$. Denote by $I^{\left(p^{\prime}\right)}=I_{r}$ the $r$ th Frobenius power, the ideal sheaf generated by the $p^{r}$ th powers of the sections of $I$. Then the $I_{r}$ and the ordinary powers $I^{r}$ define the same topology on $\boldsymbol{O}_{\mathbf{P}_{n} \times \mathbf{P}_{n}}$, hence the same completion. Denote by $\left(\mathbf{P}_{n} \times \mathbf{P}_{n}\right)_{r}$ the $r$ th Frobenius neighborhood of $\Delta$ in $\mathbf{P}_{n} \times \mathbf{P}_{n}$, whose underlying space is $\Delta$ and whose structure sheaf is 


$$
\theta_{r}=\theta_{\mathbf{P}_{n} \times \mathbf{P}_{n}} / I_{r}
$$

Thus we have

$$
\mathbf{P}_{n} \hat{\times} \mathbf{P}_{n}=\text { ind } \lim \left(\mathbf{P}_{n} \times \mathbf{P}_{n}\right)_{r} \text {, }
$$

and

$$
\hat{\theta}(\nu, \nu)=\underset{r}{\operatorname{proj}} \lim \theta(\nu, \nu) \otimes \theta_{r} .
$$

By passage to the limit with the Mittag-Leffler condition ML [EGA, $0_{\text {III }}$. 13.2 , ff.] we have a canonical isomorphism

$$
H^{i}\left(\left(\mathbf{P}_{n} \hat{\times} \mathbf{P}_{n}\right), \hat{\mathcal{O}}(\nu, \nu)\right) \approx \underset{r}{\operatorname{proj}} \lim H^{i}\left(\left(\mathbf{P}_{n} \times \mathbf{P}_{n}\right)_{r}, \theta(\nu, \nu) \otimes \theta_{r}\right) .
$$

Hence to prove (*) it suffices to show that we have

$$
H^{i}\left(\left(\mathbf{P}_{n} \times \mathbf{P}_{n}\right)_{r}, \theta(\nu, \nu) \otimes \theta_{r}\right)=0
$$

for $i=1, \ldots, n-1$, all $r \geqslant 1$.

Let $\pi: \mathbf{P}_{n} \times \mathbf{P}_{n} \rightarrow \mathbf{P}_{n} \times \mathbf{P}_{n}$ be the Frobenius morphism on $\mathbf{P}_{n} \times \mathbf{P}_{n}$. ( $\pi$ is the identity map on points, $p$ th power map on functions.) Let $\pi^{r}$ denote the $r$-fold iterate of $\pi$. Then we have

$$
\theta_{r} \cong\left(\pi^{r}\right) * \theta_{\Delta} .
$$

The projection formula ( $\pi^{r}$ is finite) then yields a canonical isomorphism

$$
H^{i}\left(\left(\mathbf{P}_{n} \times \mathbf{P}_{n}\right)_{r}, \mathcal{O}(\nu, \nu) \otimes \mathcal{O}_{r}\right) \cong H^{i}\left(\Delta, \pi_{*}^{r}(\mathcal{\theta}(\nu, \nu)) \otimes \theta_{\Delta}\right) .
$$

Let now $i=1, \ldots, n-1$. If $\pi_{*}^{r}(\theta(\nu, \nu))$ is a direct sum of line bundles of the form $\theta(\mu, \nu)$, then the right-hand side above would vanish, by the Künneth formula. (Indeed, line bundles on $\mathbf{P}_{n}$ can have nonzero cohomology only in degrees $0, n$.) Now for $\mathbf{P}_{n}$ instead of the product the analogous assertion holds [9, Corollary 6.4, p. 138]. Hence (**) holds on $\mathbf{P}_{n} \times \mathbf{P}_{n}$, since we have

$$
\pi_{*}(\theta(\nu, \nu)) \simeq p_{1}^{*}\left(\bar{\pi}_{*} \theta_{\mathbf{P}_{n}}(\nu)\right) \otimes p_{2}^{*}\left(\bar{\pi}_{*} \theta_{\mathbf{P}_{n}}(\nu)\right),
$$

again by the Künneth formula, where $\bar{\pi}$ denotes the Frobenius morphism on $\mathbf{P}_{n}$.

COROLlary (2.2.2) (GIeSEKER [4]). Every stratified vector bundle on $\mathbf{P}_{n}$ is trivial.

Proof. Combine (a), [9, Theorem 3.4, p. 96] and (1.7.2).

REMARKS. (1) I am grateful to Hartshorne for pointing out (b) and its proof.

(2) $\mathbf{P}^{n} \times \mathbf{P}^{n}-\Delta$ therefore continues the list [10] of open varieties whose cohomological dimension drops when reduced $\bmod p$.

(2.3) Preserving our earlier notations, let $F$ be a coherent sheaf on $\mathbf{P}_{n} \times \mathbf{P}_{n}$. Define $\operatorname{hd}(F)$ to be the maximum of the homological dimensions of all the 
stalks of $F$, taken over the corresponding local rings of $\mathbf{P}_{n} \times \mathbf{P}_{n}$.

Theorem (2.3.1). Let $F$ be a coherent sheaf on $\mathbf{P}_{n} \times \mathbf{P}_{n}$. Let $\hat{F}$ be the formal completion of $F$ along $\Delta$. Suppose $\operatorname{char}(k)=p>0$. Then the natural map

$$
H^{i}\left(\mathbf{P}_{n} \times \mathbf{P}_{n}, F\right) \rightarrow H^{i}\left(\mathbf{P}_{n} \hat{\times} \mathbf{P}_{n}, \hat{F}\right)
$$

is bijective for all $i<n-\operatorname{hd}(F)$, and injective for $i=n-\operatorname{hd}(F)$.

Proof. First suppose hd $(F)=0$. Then $F$ is locally free. By $[9$, Theorem 3.4, p. 96] our assertion is equivalent to assertion (c) of (2.2.1), and thus holds. If $h d(F)$ is $>0$ we can proceed by induction, using a resolution

$$
0 \rightarrow K \rightarrow \underset{\text { finite }}{\oplus} \theta(-\nu,-\nu) \rightarrow F \rightarrow 0,
$$

which exists because $F \otimes \theta(\nu, \nu)$ is spanned by its global sections for $\nu \gg 0$. Comparing the cohomology of this sequence with that of its completion, we can apply the case hd $=0$ to the terms involving $\theta(-\nu,-\nu)$ and induction to those involving $K$. Our statement follows from the "long" 5-lemma, applied to the resulting commutative ladder.

3. Intersections. In (3.1) we develop our "intersection theory" for the condition $S(X, Y)$. As a consequence we obtain in (3.2) the main result (3.2.1), which states that if $Y$ is an $s$-dimensional Cohen-Macaulay subscheme of $\mathbf{P}_{n}$, over an algebraically closed field of characteristic $p>0$, with

$$
s \geqslant \frac{1}{2}(n+1) \text {, }
$$

then every stratified vector bundle on $Y$ is trivial.

Throughout this section we shall use the following notations: $k$ will be an algebraically closed field, $X$ will be a smooth projective $k$-variety with fixed very ample invertible sheaf $\theta_{X}(1)$, and $V$ and $Z$ will denote closed subschemes of $X$. Except where the results of (2.3) are used, our proofs are independent of the characteristic.

(3.1) For $V, Z \subset X$ as above, denote by $\hat{X}$ the formal completion of $X$ along $Z$, by $\hat{V}$ the formal completion of $V$ along $Z \cap V$. Then by [EGA I, 10.9.9] the diagram

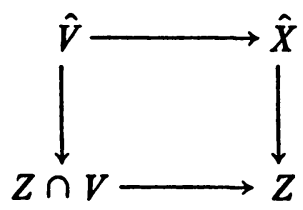

is a fibre product in the category of formal schemes. Let

$$
I=I_{V} \subset \theta_{x}
$$

be the sheaf of ideals defining $V$, and let 


$$
\hat{I}=\hat{I}_{/ z} \subset \theta_{\hat{x}}
$$

be its completion along $Z$. Since (3.1.1) is a fibre product, it follows that the sequence

$$
0 \rightarrow \hat{I} \rightarrow \theta_{\hat{x}} \rightarrow \theta_{\hat{V}} \rightarrow 0
$$

is exact, for $\hat{I}$ defines the fibre product

$$
(Z \cap V) \times{ }_{z} \hat{X} \rightarrow \hat{X}
$$

of formal schemes. Twisting $\nu$ times with $\theta_{X}(1)$ we therefore obtain an exact commutative ladder

$$
\begin{aligned}
& 0 \rightarrow \hat{I}(\nu) \rightarrow \theta_{\hat{x}}(\nu) \rightarrow \theta_{\hat{\nu}}(\nu) \rightarrow 0 \\
& \begin{array}{c}
\uparrow \\
0 \rightarrow I(\nu)
\end{array} \rightarrow \theta_{X}^{\uparrow}(\nu) \rightarrow \theta_{V}^{\uparrow}(\nu) \rightarrow 0
\end{aligned}
$$

TheOREM (3.1.1) Suppose, with $X, Z$ and $V$ as above, that $S(X, Z)$ holds. If also

$$
H^{1}(\hat{X}, \hat{I}(\nu))=0
$$

for $\nu \gg 0$, then $S(V, Z \cap V)$ holds.

Proof. We have to show that the natural map $H^{0}(V, F) \rightarrow H^{0}(\hat{V}, \hat{F})$ is bijective for any locally free coherent sheaf $F$ on $V$. We consider $F=\theta(\nu)$ for $\nu \gg 0$ first, then general $F$. By the discussion above we have a commutative diagram with exact rows

$$
\begin{aligned}
& 0 \rightarrow H^{0}(\hat{X}, \hat{I}(\nu)) \rightarrow H^{0}\left(\hat{X}, \mathcal{O}_{\hat{X}}(\nu)\right) \rightarrow H^{0}\left(\hat{V}, \mathcal{O}_{\hat{\nu}}(\nu)\right) \rightarrow H^{1}(\hat{X}, \hat{I}(\nu)) \\
& \uparrow \alpha \quad \approx \uparrow \beta \quad \uparrow \gamma \quad \uparrow \\
& 0 \rightarrow H^{0}(X, I(\nu)) \rightarrow H^{0}\left(X, \theta_{X}(\nu)\right) \rightarrow H^{0}\left(V, \theta_{V}(\nu)\right) \rightarrow H^{1}(X, I(\nu))
\end{aligned}
$$

Applying Krull's intersection theorem to the stalks of the coefficient sheaves, it follows that $\alpha, \beta$ and $\gamma$ are injective, and $\beta$ is of course bijective by $S(X, Z)$. To show $\gamma$ is bijective for $\nu \gg 0$ it suffices to show that $\gamma$ is surjective for $\nu \gg 0$. Take $\nu$ so large that $H^{1}(\hat{X}, \hat{I}(\nu))=0$ : then $\gamma$ is surjective by an obvious diagram chase.

Let now $F$ be an arbitrary locally free sheaf of finite rank on $V$. We can resolve the dual sheaf $F^{V}$ on the left by coherent sheaves of the form $\oplus \theta_{V}(-v)$ for $\nu \gg 0$. This gives an exact sequence

$$
\oplus \mathcal{O}_{V}\left(-\nu^{\prime}\right) \rightarrow \oplus \mathcal{O}_{V}(-\nu) \rightarrow F^{V} \rightarrow 0 .
$$

Passing to the dual we obtain a resolution on the right:

$$
0 \rightarrow F \rightarrow \oplus \mathcal{O}_{\nu}(v) \rightarrow \oplus \mathcal{O}_{V}\left(\nu^{\prime}\right) .
$$

Completing along $Z \cap V$ and taking $H^{0}$, which preserves kernels, we obtain a commutative diagram with exact rows 


$$
\begin{aligned}
& 0 \rightarrow H^{0}(\hat{V}, \hat{F}) \rightarrow \oplus H^{0}\left(\hat{V}, \theta_{\hat{\nu}}(\nu)\right) \rightarrow \oplus H^{0}\left(\hat{V}, \theta_{\hat{\nu}}\left(\nu^{\prime}\right)\right) \\
& \uparrow \alpha \quad \uparrow \beta \quad \uparrow \gamma \\
& 0 \rightarrow H^{0}(V, F) \rightarrow \oplus H^{0}\left(V, \theta_{V}(\nu)\right) \rightarrow \oplus H^{0}\left(V, \theta_{V}\left(\nu^{\prime}\right)\right)
\end{aligned}
$$

Since $\nu$ and $\nu^{\prime}$ can be as large as we like, we can arrange things so that $\beta$ and $\gamma$ are bijective, by the first part of the proof. Then $\alpha$ is bijective too, by the universal nature of kernels. Hence $S(V, Z \cap V)$ holds, and our proof is complete.

CoRollaRY (3.1.2) Let $Y \subset \mathbf{P}_{n}$ be a pure s-dimensional Cohen-Macaulay subscheme. Assume the characteristic is $p>0$ and that

$$
s \geqslant \frac{1}{2}(n+1) \text {. }
$$

Then $S(Y \times Y, \Delta(Y))$ holds.

Proof. Let $I \subset \mathbf{P}_{n} \times \mathbf{P}_{n}$ be the ideal sheaf defining $Y \times Y$ in $\mathbf{P}_{n} \times \mathbf{P}_{n}$. From the exact sequence

$$
0 \rightarrow I \rightarrow \theta_{\mathbf{P}_{n} \times \mathbf{P}_{n}} \rightarrow \hat{\theta}_{Y \times Y} \rightarrow 0
$$

we find

$$
\operatorname{hd}(I)=\operatorname{hd}\left(\theta_{Y \times Y}\right)-1_{1}=(2 n-2 s)-1 .
$$

Next we claim that $H^{1}\left(\mathbf{P}_{n} \times \mathbf{P}_{n}, \hat{I}(\nu)\right)=0$ for any $\nu$. For this we can use (2.3.1), so we only need to show that $n-\operatorname{hd}(I)$ is $>1$. But since we have

$$
n-\operatorname{hd}(I)=2 s-n+1
$$

by the above, $s>\frac{1}{2}(n+1)$ yields $n-\operatorname{hd}(I)>1$ as desired.

Since $H^{\mathrm{I}}\left(\mathbf{P}_{n} \times \mathbf{P}_{n}, \hat{I}(\nu)\right)=0$, we can apply (3.1.3) with

$$
V=Y \times Y, \quad Z=\Delta \subset X=\mathbf{P}_{n} \times \mathbf{P}_{n} .
$$

We conclude that $S(Y \times Y, \Delta(Y))$ holds, and this completes the proof.

(3.2) We now turn our attention to stratified bundles. Combining (1.7.1) and (3.1.2) we obtain

THEOREM (3.2.1) (TRIVIALITY OF STRATIFIED BUNDLES). Let $k$ be an algebraically closed field of characteristic $p>0$, and let $Y \subset \mathbf{P}_{n}$ be a closed Cohen-Macaulay subscheme of dimension s, such that we have

$$
s>\frac{1}{2}(n+1) \text {. }
$$

Then every stratified vector bundle on $Y$ is trivial.

(3.3) The main result (3.1.2) also yields the following application to the theory of cohomological dimension.

Theorem (3.3.1) (AMPLENesS OF THE DiAgonal). Hypotheses as in (3.2.1), then

$$
\operatorname{cd}(Y \times Y-\Delta(Y))<2 \operatorname{dim}(Y)-2,
$$

and 


$$
\operatorname{Lef}(Y \times Y, \Delta(Y))
$$

holds.

Proof. Apply [9, Theorem 3.4, p. 96].

REMARK. Hartshorne has suggested [9, p. 89] that, for $n>1, \mathbf{P}_{n}$ may be the only smooth projective variety with ample tangent bundle, and has verified this for $n<2$. Hence one should not expect $\Delta(Y) \subset Y \times Y$ to have ample normal bundle if $Y \neq \mathbf{P}_{n}$. It is pleasant, therefore, to discover that the diagonal embedding of a variety $Y$ of low codimension has so many related properties.

4. The fundamental group. We review the concept of principal parts in (4.1) and then obtain our main theorem (4.2.1) for the fundamental group. (4.1) includes a fine lemma due to Altman and Kleiman, and the proof of (4.2.1) is based on an idea first published by Gieseker [4].

(4.1) Let $k$ be a ring, $X$ a $k$-scheme. As before, we form the product $X \times X$ over $\operatorname{Spec}(k)$, and let $\Delta \subset X \times X$ be the diagonal. Let

$$
I \subset \theta_{X \times X}
$$

be the ideal sheaf defining $\Delta$. Thus the $r$ th infinitesimal neighborhood of $\Delta$ in $X \times X$ is

$$
\Delta_{r}=\operatorname{Spec}\left(\theta_{X \times X} / I^{r+1}\right) .
$$

We define the sheaf of principal parts of order $r$ of $X$ to be

$$
\operatorname{Pr}^{r}(X)=\theta_{X \times X} / I^{r+1} \text {. }
$$

We view this as an $\theta_{X}$-bimodule via the left and right structures of $\theta_{X \times X}=$ $\theta_{x} \otimes_{k} \theta_{x}$.

Let now $f: X \rightarrow Y$ be an étale covering of $k$-schemes. We are going to define two maps

$$
\eta_{R}, \eta_{L}: f^{*}(\mathcal{P} r(Y)) \rightarrow \mathscr{P r}(X)
$$

Over a suitable affine on $Y$, the principal parts are generated by tensors $a_{1} \otimes a_{2}$, where $a_{1}, a_{2}$ are sections of $\theta_{Y}$. Denote by $s$ a section of $\theta_{X}$. Then we define $\eta_{R}$ via

$$
\left(a_{1} \otimes a_{2}\right) \otimes s \rightarrow a_{1} \otimes a_{2} s .
$$

Hence $\eta_{R}$ acts on the right structures of $\mathscr{P r}(Y)$ and $\mathscr{P r}(X)$. We define $\eta_{L}$ via the assignment

$$
s \otimes\left(a_{1} \otimes a_{2}\right) \rightarrow s a_{1} \otimes a_{2},
$$

so that $\eta_{L}$ acts on the left structures.

Lemma (4.1) (Altman-Kleiman). Notations as above, with $f: X \rightarrow Y$ étale. Then $\eta_{L}$ and $\eta_{R}$ are isomorphisms 


$$
f^{*}(\mathcal{P r}(Y)) \cong \operatorname{Pr}(X) \text {. }
$$

Proof. We reduce immediately to the case of $X, Y$ affine, and this is precisely [1, Lemma 1, p. 463].

(4.2) Here is our second main result:

TheOREM (4.2.1) (CONNECTEDNess). Let $Y$ be an s-dimensional CohenMacaulay subscheme of $\mathbf{P}_{n}$, over an algebraically closed base field $k$ of characteristic $p>0$. Suppose

$$
s \geqslant \frac{1}{2}(n+1) .
$$

Then $Y$ is simply connected.

The following lemma was first established by Gieseker [4, Theorem 1.10], using his own definition of stratification. Together with (3.2.1) it immediately implies our theorem.

LemMa (4.2.1.1) With $X$ as in (4.2.1), suppose every stratified vector bundle on $X$ is trivial. Then $X$ is simply connected.

Proof. Let $f: X^{\prime} \rightarrow X$ be an étale cover. We can assume both $X^{\prime}$ and $X$ are connected, and then it suffices to show $f$ is an isomorphism.

For each $r$, the identity map

$$
\operatorname{Pr}\left(X^{\prime}\right) \stackrel{\theta_{r}}{\rightarrow} \operatorname{Pr}\left(X^{\prime}\right)
$$

defines the trivial stratification on $\theta_{X^{\prime}}$. By (4.1.1) we have

$$
\left.\mathscr{P r}\left(X^{\prime}\right) \simeq \mathcal{O}_{X^{\prime}} \otimes_{\theta_{X}} \operatorname{Pr}(X) \quad \text { (by } \eta_{L}\right)
$$

and

$$
\left.\mathscr{P r}\left(X^{\prime}\right) \simeq \mathscr{P r}(X) \otimes_{\Theta_{X}} \Theta_{X^{\prime}} \quad \text { (by } \eta_{R}\right) .
$$

Hence $\theta_{r}$ can be rewritten

$$
\theta_{X^{\prime}} \otimes_{\theta_{X}} \operatorname{Pr}(X) \stackrel{\theta_{r}}{\rightarrow} \operatorname{Pr}(X) \otimes_{\theta_{X}} \theta_{X^{\prime}},
$$

and this map is plainly $\mathcal{P r}(X)$-linear.

It is easy to see that $\theta_{r}$ therefore defines a stratification on $f_{*}\left(\theta_{X^{\prime}}\right)$, which is a vector bundle on $X$ because $f$ is finite and étale. Our main assertion will plainly follow if also $\operatorname{rank}\left(f_{*}\left(\theta_{X^{\prime}}\right)\right)=1$. Now the Leray spectral sequence for $f$ degenerates, yielding an isomorphism

$$
k=H^{0}\left(X^{\prime}, \Theta_{X^{\prime}}\right) \cong H^{0}\left(X, f_{*}\left(\Theta_{X^{\prime}}\right)\right) .
$$

Since $f_{*}\left(\theta_{x^{\prime}}\right)$ is a trivial vector bundle, we find

$$
\operatorname{rank}\left(f_{*}\left(\theta_{X^{\prime}}\right)\right)=\operatorname{dim}_{k} H^{0}\left(X, f_{*}\left(\theta_{X^{\prime}}\right)\right)=1,
$$

so our proof is complete. 
REMARK. By results of Gieseker [4], it follows from (3.2.1) that $\operatorname{Pic}(X)$ has no torsion prime to $p$ if every stratified bundle on $X$ is trivial. For $X$ smooth of low codimension in characteristic 0 , however, Ogus has shown that $\operatorname{Pic}(X)$ is $\mathbf{Z}$. Therefore in characteristic $p>0$ one might expect something similar, e.g. modulo $p$-torsion. The extension of our result on $\pi_{1}$, if possible, to the Picard group would seem to be the next main task for research in this area.

\section{REFERENCES} $461 \mathrm{ff}$.

1. A. Altman and S. Kleiman, On the purity of the branch locus, Compositio Math. 23 (1971),

2. W. Barth, Transplanting cohomology classes in complex projective space, Amer. J. Math. 12 (1973), $951 \mathrm{ff}$.

3. W. Barth and M. Larsen, On the homotopy groups of complex projective manifolds, Math. Scand. 30 (1972), 88 ff.

4. D. Gieseker, Flat bundles and the fundamental group (to appear).

5. A. Grothendieck et al., Dix exposés sur la cohomologie des schémas, North-Holland, Amsterdam, 1968.

6. _ Revêtements etales et groupe fondamentale, Lecture Notes in Math., vol. 224, Springer, Berlin and New York, 1971.

7. Cohomologie locale des faiseaux coherénts et théorèmes de Lefschetz locaux et globaux, North-Holland, Amsterdam, 1968.

8. R. Hartshorne, Varieties of small codimension in projective space, Bull. Amer. Math. Soc. 80 (1974), 1017-1032.

9. __ Ample subvarieties of algebraic varieties, Lecture Notes in Math., vol. 156, Springer, Berlin and New York, 1970.

10. R. Hartshorne and R. Speiser, Local cohomological dimension in characteristic p, Ann. of Math. (2) 105 (1977), 45-79.

11. A. Ogus, Local cohomological dimension of algebraic varieties, Ann. of Math. (2) 98 (1973), $327 \mathrm{ff}$.

12. __ On the formal neighborhood of a subvariety of projective space (to appear).

13. J.-P. Serre, Faisceaux algébriques cohérents, Ann. of Math. (2) 61 (1955), $197 \mathrm{ff.}$

14. R. Speiser, Cohomological dimension of noncomplete hypersurfaces, Invent. Math. 21 (1973), $143 \mathrm{ff}$.

15. __, Cohomological dimension and Abelian varieties, Amer. J. Math. 95 (1973), 1-34.

Department of Mathematics, Illinois State University, Normal, Illinois 61701 